\title{
Electrovacuum Static Counterrotating Relativistic Dust Disks
}

\author{
Gonzalo García R* and Guillermo A. Gonzáleđđ \\ Escuela de Física, Universidad Industrial de Santander, A. A. 678, Bucaramanga, Colombia
}

\begin{abstract}
A detailed study is presented of the counterrotating model (CRM) for generic electrovacuum static axially symmetric relativistic thin disks without radial pressure. We find a general constraint over the counterrotating tangential velocities needed to cast the surface energy-momentum tensor of the disk as the superposition of two counterrotating charged dust fluids. We also find explicit expressions for the energy densities, charge densities and velocities of the counterrotating fluids. We then show that this constraint can be satisfied if we take the two counterrotating streams as circulating along electrogeodesics. However, we show that, in general, it is not possible to take the two counterrotating fluids as circulating along electro-geodesics nor take the two counterrotating tangential velocities as equal and opposite. Four simple families of models of counterrotating charged disks based on ChazyCurzon-like, Zipoy-Voorhees-like, Bonnor-Sackfield-like and Kerr-like electrovacuum solutions are considered where we obtain some disks with a CRM well behaved. The models are constructed using the well-known "displace, cut and reflect" method extended to solutions of vacuum EinsteinMaxwell equations.
\end{abstract}

PACS numbers: 04.20.-q, 04.20.Jb, 04.40.Nr

\section{INTRODUCTION}

Stationary or static axially symmetric exact solutions of Einstein equations describing relativistic thin disks are of great astrophysical importance since can be used as models of certain stars, galaxies and accretion disks. These were first studied by Bonnor and Sackfield [1], obtaining pressureless static disks, and then by Morgan and Morgan, obtaining static disks with and without radial pressure [2, 3]. In connection with gravitational collapse, disks were first studied by Chamorro, Gregory and Stewart [4]. Disks with radial tension have been also studied [5]. Several classes of exact solutions of the Einstein field equations corresponding to static and stationary thin disks have been obtained by different authors [6, 7, 8, 9, 10, 11, 12, 13, 14, 15, 16], with or without radial pressure.

In the case of static disks without radial pressure, there are two common interpretations. The stability of these models can be explained by either assuming the existence of hoop stresses or that the particles on the disk plane move under the action of their own gravitational field in such a way that as many particles move clockwise as counterclockwise. This last interpretation, the "counterrotating model" (CRM), is frequently made since it can be invoked to mimic true rotational effects. Even though this interpretation can be seen as a device, there are observational evidence of disks made of streams of rotating and counterrotating matter [17, 18].

Disk sources for stationary axially symmetric spacetimes with magnetic fields are also of astrophysical importance mainly in the study of neutron stars, white dwarfs and galaxy formation. Although disks with electric fields do not have clear astrophysical importance, their study may be of interes in the context of exact solutions. Thin disk have been discussed as sources for Kerr-Newman fields [19], magnetostatic axisymmetric fields [20] and conformastationary metrics [21]. Following the Ref. [19] the resultating disks can be interpreted either as rings with internal pressure and currents or as two counterrotating streams of freely moving charged particles, i.e. which move along electro-geodesics (solution to the geodesic equation in the presence of a Lorentz force).

In all the above cases, the disks are obtained by an "inverse problem" approach, called by Synge the "g-method" [22]. The method works as follows: a solution of the vacuum Einstein equations is taken, such that there is a discontinuity in the derivatives of the metric tensor on the plane of the disk, and the energy-momentum tensor is obtained from the Einstein equations. The physical properties of the matter distribution are then studied by an analysis of the surface energy-momentum tensor so obtained. On the other hand, a "direct problem" approach, called by Synge the " $T$ method", is also used by other authors [23, 24, 25, 26, 27, 28, 29] by taking a given surface energy-momentum tensor and solving the Einstein equations in the matter region. The inner solution is then used to obtain boundary data for the vacuum field equations in the outer region. The T-method is used in Ref. 23 to obtain the solution to the problem of a (one-component) uniformly rotating disk of dust, and in Refs. 24, 25, 26, 27, 28, 29] to generate counterrotating

*e-mail: ggr1970@yahoo.com

†e-mail: guillego@uis.edu.co 
dust disks, but no condition is imposed there about the (electro-) geodesic motion of the two counterrotating streams.

The aim of this paper is to perform a detailed study of the CRM for generic electrovacuum static axially symmetric relativistic thin disks without radial pressure. The counterrotating model for the case of static thin disks without electric or magnetic fields was recently studied in [30], so the material presented here is a continuation of the mentioned work. The paper is organized as follows. In Sec. II we present a summary of the procedure to obtain thin disks models with a purely azimuthal pressure and currents, using the well-known "displace, cut and reflect" method extended to solutions of Einstein-Maxwell equations. In particular, we obtain expressions for the surface energy-momentum tensor and the current density of the disks.

Next, in Sec. III, the disks are interpreted in terms of the CRM. We find a general constraint over the counterrotating tangential velocities needed to cast the surface energy-momentum tensor of the disk as the superposition of two counterrotating charged dust fluids. We also find explicit expressions for the energy densities, current densities and velocities of the counterrotating fluids. We then show that this constraint can be satisfied if we take the two counterrotating streams as circulating along electro-geodesics. However, we show that, in general, it is not possible to take the two counterrotating fluids as circulating along electro-geodesics nor take the two counterrotating tangential velocities as equal and opposite.

In the following section, Sec. IV, four simple families of models of counterrotating charged disks based on ChazyCurzon-like, Zipoy-Voorhees-like, Bonnor-Sackfield-like and Kerr-like metrics are presented where we obtain some disks with a CRM well behaved. In particular, we study the tangential velocities, mass and electric charge densities of both streams in the pure static and electrostatic (magnetostatic) cases. Also the stability against radial perturbation is analyzed. Finally, in Sec. V, we summarize our main results.

\section{ELECTROVACUUM STATIC RELATIVISTIC DISKS}

In this section we present a summary of the procedure to obtain electrovacuum static axially symmetric relativistic thin disks. The simplest metric to describe a static axially symmetric spacetime is the Weyl's line element

$$
d s^{2}=-e^{2 \nu} d t^{2}+e^{-2 \nu}\left[r^{2} d \varphi^{2}+e^{2 \lambda}\left(d r^{2}+d z^{2}\right)\right]
$$

where $\nu$ and $\lambda$ are functions of $r$ and $z$ only. The vacuum Einstein-Maxwell equations, in geometrized units such that $8 \pi G=c=\mu_{0}=\varepsilon_{0}=1$, are given by

$$
\begin{aligned}
& R_{a b}=T_{a b}, \\
& T_{a b}=F_{a c} F_{b}^{c}-\frac{1}{4} g_{a b} F_{c d} F^{c d}, \\
& F_{; b}^{a b}=0 \\
& F_{a b}=A_{b, a}-A_{a, b},
\end{aligned}
$$

where all symbols are understood.

For the metric (1), the Einstein-Maxwell equations in vacuum are equivalent the complex Ernst equations [31]

$$
\begin{aligned}
& f \Delta \mathcal{E}=\left(\nabla \mathcal{E}+2 \Phi^{*} \nabla \Phi\right) \cdot \nabla \mathcal{E}, \\
& f \Delta \Phi=\left(\nabla \mathcal{E}+2 \Phi^{*} \nabla \Phi\right) \cdot \nabla \Phi,
\end{aligned}
$$

with $\mathcal{E}=\mathcal{E}^{*}$ (static spacetime), where $\Delta$ and $\nabla$ are the standard differential operators in cylindrical coordinates and $f=e^{2 \nu}$. The metric functions are obtained via

$$
\begin{aligned}
f & =\mathcal{E}+\Phi \Phi^{*} \\
\lambda_{, \zeta} & =\frac{\sqrt{2} r}{4 f^{2}}\left(\mathcal{E}_{, \zeta}+2 \Phi^{*} \Phi_{, \zeta}\right)\left(\mathcal{E}_{, \zeta}+2 \Phi \Phi_{, \zeta}^{*}\right)-\frac{\sqrt{2} r}{f} \Phi_{, \zeta} \Phi_{, \zeta}^{*}
\end{aligned}
$$


where $\sqrt{2} \zeta=r+i z$, so that $\sqrt{2} \partial_{, \zeta}=\partial_{, r}-i \partial_{, z}$. The electromagnetic potencials are related to $\Phi$ via

$$
\begin{aligned}
A_{t} & =\sqrt{2} \operatorname{Re} \Phi, \\
A_{\varphi, \zeta} & =\sqrt{2} i \frac{r}{f}(\operatorname{Im} \Phi)_{, \zeta} .
\end{aligned}
$$

In order to obtain a solution of (3) representing a thin disk at $z=0$, we assume that the components of the metric tensor are continuous across the disk, but their first derivates discontinuous on the plane $z=0$, with discontinuity functions

$$
b_{a b}=\left.g_{a b, z}\right|_{z=0^{+}}-\left.g_{a b, z}\right|_{z=0^{-}}=\left.2 g_{a b, z}\right|_{z=0^{+}} .
$$

Thus, the Einstein-Maxwell equations yield an energy-momentum tensor $T_{a}^{b}=Q_{a}^{b} \delta(z)$ and a planar current density $\mathrm{J}_{a}=j_{a} \delta(z)=-2 A_{a, z} \delta(z)$, where $\delta(z)$ is the usual Dirac function with support on the disk and

$$
Q_{b}^{a}=\frac{1}{2}\left\{b^{a z} \delta_{b}^{z}-b^{z z} \delta_{b}^{a}+g^{a z} b_{b}^{z}-g^{z z} b_{b}^{a}+b_{c}^{c}\left(g^{z z} \delta_{b}^{a}-g^{a z} \delta_{b}^{z}\right)\right\}
$$

is the distributional energy-momentum tensor. The "true" surface energy-momentum tensor (SEMT) of the disk, $S_{a}^{b}$, can be obtained through the relation

$$
S_{a}^{b}=\int T_{a}^{b} d s_{n}=e^{\lambda-\nu} Q_{a}^{b}
$$

where $d s_{n}=\sqrt{g_{z z}} d z$ is the "physical measure" of length in the direction normal to the disk, and the surface current density as $\mathrm{j}_{a}=e^{\lambda-\nu} j_{a}$. For the metric (10), the non-zero components of $S_{a}^{b}$ and the current density are

$$
\begin{aligned}
& S_{0}^{0}=2 e^{\nu-\lambda}\{\lambda, z-2 \nu, z\}, \\
& S_{1}^{1}=2 e^{\nu-\lambda} \lambda, z,
\end{aligned}
$$

and

$$
\begin{aligned}
& \mathrm{j}_{t}=-2 e^{\nu-\lambda} A_{t, z}, \\
& \mathrm{j}_{\varphi}=-2 e^{\nu-\lambda} A_{\varphi, z},
\end{aligned}
$$

where all the quantities are evaluated at $z=0^{+}$.

With an orthonormal tetrad $\mathrm{e}_{\hat{a}}^{b}=\left\{V^{b}, W^{b}, X^{b}, Y^{b}\right\}$, where

$$
\begin{aligned}
V^{a} & =e^{-\nu}(1,0,0,0), \\
W^{a} & =\frac{e^{\nu}}{r}(0,1,0,0), \\
X^{a} & =e^{\nu-\lambda}(0,0,1,0), \\
Y^{a} & =e^{\nu-\lambda}(0,0,0,1),
\end{aligned}
$$

we can write the metric and the SEMT in the canonical forms

$$
\begin{aligned}
& g_{a b}=-V_{a} V_{b}+W_{a} W_{b}+X_{a} X_{b}+Y_{a} Y_{b}, \\
& S_{a b}=\epsilon V_{a} V_{b}+p_{\varphi} W_{a} W_{b},
\end{aligned}
$$

where

$$
\epsilon=-S_{0}^{0} \quad, \quad p_{\varphi}=S_{1}^{1},
$$

are, respectively, the energy density and the azimuthal pressure of the disk. 


\section{THE COUNTERROTATING MODEL}

We now consider, based on references [32] and [33], the possibility that the SEMT $S^{a b}$ and the current density $\mathrm{j}^{a}$ can be written as the superposition of two counterrotating fluids that circulate in opposite directions; that is, we assume

$$
\begin{aligned}
S^{a b} & =S_{+}^{a b}+S_{-}^{a b}, \\
\mathrm{j}^{a} & =\mathrm{j}_{+}^{a}+\mathrm{j}_{-}^{a},
\end{aligned}
$$

where the quantities in the right-hand side are, respectively, the SEMT and the current density of the prograde and retrograde counterrotating fluids.

Let $U_{ \pm}^{a}=\left(U_{ \pm}^{0}, U_{ \pm}^{1}, 0,0\right)$ be the velocity vectors of the two counterrotating fluids. In order to do the decomposition (12a) and (12b) we project the velocity vectors onto the tetrad $\mathrm{e}_{\hat{a}}{ }^{b}$, using the relations [34]

$$
U_{ \pm}^{\hat{a}}=\mathrm{e}^{\hat{a}}{ }_{b} U_{ \pm}^{b} \quad, \quad U_{ \pm}^{a}=U_{ \pm}^{\hat{c}} \mathrm{e}_{\hat{c}}^{a} .
$$

With the tetrad (9) we can write

$$
U_{ \pm}^{a}=\frac{V^{a}+\mathrm{U}_{ \pm} W^{a}}{\sqrt{1-\mathrm{U}_{ \pm}^{2}}}
$$

and thus

$$
\begin{aligned}
V^{a} & =\frac{\sqrt{1-\mathrm{U}_{-}^{2}} \mathrm{U}_{+} U_{-}^{a}-\sqrt{1-\mathrm{U}_{+}^{2}} \mathrm{U}_{-} U_{+}^{a}}{\mathrm{U}_{+}-\mathrm{U}_{-}}, \\
W^{a} & =\frac{\sqrt{1-\mathrm{U}_{+}^{2}} U_{+}^{a}-\sqrt{1-\mathrm{U}_{-}^{2}} U_{-}^{a}}{\mathrm{U}_{+}-\mathrm{U}_{-}},
\end{aligned}
$$

where $\mathrm{U}_{ \pm}=U_{ \pm}^{\hat{1}} / U_{ \pm}^{\hat{0}}$ are the tangential velocities of the fluids with respect to the tetrad.

Using (15), we can write the SEMT as

$$
\begin{aligned}
S^{a b}= & \frac{f\left(\mathrm{U}_{-}, \mathrm{U}_{-}\right)\left(1-\mathrm{U}_{+}^{2}\right) U_{+}^{a} U_{+}^{b}}{\left(\mathrm{U}_{+}-\mathrm{U}_{-}\right)^{2}}+\frac{f\left(\mathrm{U}_{+}, \mathrm{U}_{+}\right)\left(1-\mathrm{U}_{-}^{2}\right) U_{-}^{a} U_{-}^{b}}{\left(\mathrm{U}_{+}-\mathrm{U}_{-}\right)^{2}} \\
& -\frac{f\left(\mathrm{U}_{+}, \mathrm{U}_{-}\right)\left[\left(1-\mathrm{U}_{+}^{2}\right)\left(1-\mathrm{U}_{-}^{2}\right)\right]^{\frac{1}{2}}\left(U_{+}^{a} U_{-}^{b}+U_{-}^{a} U_{+}^{b}\right)}{\left(\mathrm{U}_{+}-\mathrm{U}_{-}\right)^{2}}
\end{aligned}
$$

where

$$
f\left(\mathrm{U}_{1}, \mathrm{U}_{2}\right)=\epsilon \mathrm{U}_{1} \mathrm{U}_{2}+p_{\varphi}
$$

Clearly, in order to cast the SEMT in the form (12a), the mixed term must be absent and therefore the counterrotating tangential velocities must be related by

$$
f\left(\mathrm{U}_{+}, \mathrm{U}_{-}\right)=0
$$

where we assume that $\left|U_{ \pm}\right| \neq 1$. Then, assuming a given choice for the counterrotating velocities in agreement with the above relation, we can write the SEMT as (12a) with

$$
S_{ \pm}^{a b}=\epsilon_{ \pm} U_{ \pm}^{a} U_{ \pm}^{b}
$$

so that we have two counterrotating dust fluids with energy densities given by

$$
\epsilon_{ \pm}=\left[\frac{1-\mathrm{U}_{ \pm}^{2}}{\mathrm{U}_{\mp}-\mathrm{U}_{ \pm}}\right] \mathrm{U}_{\mp} \epsilon
$$


Thus the SEMT $S^{a b}$ can be written as the superposition of two counterrotating dust streams if, and only if, the constraint (17) admits a solution such that $U_{+} \neq U_{-}$. This result is completely equivalent to the necessary and sufficient condition obtained in reference [33].

Similarly, we can write the current density as (12b) with

$$
\mathrm{j}_{ \pm}^{a}=\sigma_{ \pm} U_{ \pm}^{a}
$$

where $\sigma_{ \pm}$are the counterrotating electric charge densities of the fluids which are given by

$$
\sigma_{ \pm}=\left[\frac{\sqrt{1-U_{ \pm}^{2}}}{\mathrm{U}_{ \pm}-\mathrm{U}_{\mp}}\right]\left(\frac{\mathrm{j}^{1}}{W^{1}}-\frac{\mathrm{j}^{0}}{V^{0}} \mathrm{U}_{\mp}\right) .
$$

Another quantity related with the counterrotating motion is the specific angular momentum of a particle rotating at a radius $r$, defined as $h_{ \pm}=g_{\varphi \varphi} U_{ \pm}^{\varphi}$. We can write

$$
h_{ \pm}=\frac{r e^{-\nu} \mathrm{U}_{ \pm}}{\sqrt{1-\mathrm{U}_{ \pm}^{2}}} .
$$

This quantity can be used to analyze the stability of the disks against radial perturbations. The condition of stability,

$$
\frac{d\left(h^{2}\right)}{d r}>0
$$

is an extension of the Rayleigh criteria of stability of a fluid in rest in a gravitational field [35].

As we can see from Eqs. (14), (19), (21) and (22), all the physical quantities asociated with the CRM depend of the counterrotating tangential velocities $U_{ \pm}$. However, the constraint (17) does not determine $U_{ \pm}$uniquely, and so we need to imposse some additional requeriment in order to obtain a complete determination of the tangential velocities, leading so to a well defined CRM. A possibility, commonly assumed, is to take the two counterrotating fluids as circulating along electro-geodesics

$$
\frac{1}{2} \epsilon_{ \pm} g_{a b, r} U_{ \pm}^{a} U_{ \pm}^{b}=-\sigma_{ \pm} F_{r a} U_{ \pm}^{a}
$$

The conservation laws (the Bianchi identities) at the disk then imply that the two counterrotating streams interact only by means of gravitational and electromagnetic forces.

Let $\omega_{ \pm}=U_{ \pm}^{1} / U_{ \pm}^{0}$ be the angular velocities of the particles. In terms of $\omega_{ \pm}$we get

$$
\mathrm{U}_{ \pm}=\left[\frac{V^{0}}{W^{1}}\right] \omega_{ \pm}
$$

and so, using (14), (19) and (21), (24) takes the form

$$
\omega_{\mp}\left(g_{11, r} \omega_{ \pm}^{2}+g_{00, r}\right)=-\frac{2 V_{0}^{2}}{\epsilon}\left(\mathrm{j}^{0} \omega_{\mp}-\mathrm{j}^{1}\right)\left(A_{t, r}+A_{\varphi, r} \omega_{ \pm}\right),
$$

so that

$$
\begin{aligned}
\omega_{+}+\omega_{-} & =\frac{2}{\epsilon}\left[\frac{W^{1}}{V^{0}}\right]^{2}\left[\frac{\epsilon \mathrm{j}_{1} A_{t, r}+p_{\varphi} \mathrm{j}_{0} A_{\varphi, r}}{\epsilon g_{00, r}+2 \mathrm{j}_{0} A_{t, r}}\right], \\
\omega_{+} \omega_{-} & =-\left[\frac{W^{1}}{V^{0}}\right]^{2} \frac{p_{\varphi}}{\epsilon},
\end{aligned}
$$

where we have used the Einstein-Maxwell equation (4b) and the expressions (7a $-(8 \mathrm{~b})$ for the SEMT and the current density. From the last expression follows immediately that $f\left(\mathrm{U}_{+}, \mathrm{U}_{-}\right)$vanishes and so the electro-geodesic velocities agree with (17) and we have a well defined CRM.

Note that in general the two fluids circulate with different velocities. However, when the spacetime is electrostatic (or magnetostatic) the two electro-geodesic fluids circulate with equal and opposite velocities, so that the constraint (17) is equivalent to

$$
\mathrm{U}^{2}=\frac{p_{\varphi}}{\epsilon},
$$


as is commonly assumed in the works concerning counterrotating disks. We now have two counterrotating charged dust streams with equal energy densities

$$
\epsilon_{ \pm}=\frac{\epsilon-p_{\varphi}}{2}
$$

specific angular momenta

$$
h_{ \pm}= \pm r e^{-\nu} \sqrt{\frac{p_{\varphi}}{\epsilon-p_{\varphi}}}
$$

and electric charge densities

$$
\begin{aligned}
\sigma_{e \pm} & =-\frac{1}{2} e^{-\nu} \mathrm{j}_{0} \sqrt{1-\frac{p_{\varphi}}{\epsilon}}, \\
\sigma_{m \pm} & = \pm \frac{1}{2 r} e^{\nu} \mathrm{j}_{1} \sqrt{\frac{\epsilon}{p_{\varphi}}-1},
\end{aligned}
$$

where $\sigma_{e \pm}$ and $\sigma_{m \pm}$ are the electric charge densities in the electrostatic and magnetostatic cases, respectively. The velocities are given by (27).

Although the electro-geodesic choice leads to a well defined CRM, sometimes the corresponding physical quantities may have unphysical behavior. For instance, the expressions (21) and (22) may take imaginary values. So we need to consider other solutions of Eq. (17) differents of the electro-geodesic velocities. Another possibility, commonly considered, is to take the two counterrotating fluids not circulating along electro-geodesics but with equal and opposite tangential velocities,

$$
\mathrm{U}_{ \pm}= \pm \mathrm{U}
$$

This choice, that imply the existence of additional interactions between the two streams (e.g. colllisions), leads to a complete determination of the velocity vectors in such away that the expressions for the velocities, energy densities and specific angular momenta of both streams coincide with the electrostatic (magnetostatic) case, but the charge densities are differents. However, this can be made only when $0 \leq\left|p_{\varphi} / \sigma\right| \leq 1$. If this is not the case, we cannot take the two velocities as equal and opposite. In the general case, the two counterrotating streams circulate with different velocities and we can write (17) as

$$
\mathrm{U}_{+} \mathrm{U}_{-}=-\frac{p_{\varphi}}{\epsilon} .
$$

However, this relation does not determine completely the tangential velocities, and so the CRM is undetermined.

\section{SOME SIMPLE EXAMPLES OF COUNTERROTATING CHARGED DUST DISKS}

\section{A. CRM for Chazy-Curzon-like disks}

The first family of solutions considered is a Chazy-Curzon-like solution which is given by

$$
\begin{aligned}
e^{\nu} & =\frac{2}{(1+a) e^{\gamma / \rho}+(1-a) e^{-\gamma / \rho}} \\
\lambda & =-\frac{\gamma^{2} \sin ^{2} \theta}{2 \rho^{2}} \\
A_{t} & =\frac{\sqrt{2} p\left[e^{\gamma / \rho}-e^{-\gamma / \rho}\right]}{(1+a) e^{\gamma / \rho}+(1-a) e^{-\gamma / \rho}} \\
A_{\varphi} & =\sqrt{2} \gamma q \cos \theta
\end{aligned}
$$

where $a^{2}=1+b^{2}$, with $b^{2}=p^{2}+q^{2}$, and $\gamma$ is a real constant. Here $p$ and $q$ are the electric and magnetic parameters, respectively. $\rho$ and $\theta$ are the spherical coordinates, related to the Weyl coordinates by

$$
r=\rho \sin \theta, \quad z+z_{0}=\rho \cos \theta .
$$


Note that we have displaced the origin of the $z$ axis in $z_{0}$. This solution can be generated, in these coordinates, using the well-known complex potencial formalism proposed by Ernst [31] from the Chazy-Curzon vacuum solution [36, 37], by choosing the parameter $q$ of Ref. 31] as complex. For $q=0$ we have an electrostatic solution and for $p=0$ one obtains its magnetostatic analogue [38].

From the above expressions we can compute the physical quantities associated with the disks. We obtain

$$
\begin{aligned}
\epsilon & =\frac{8 \gamma \cos \theta e^{-\lambda}\left[\left(\rho-\gamma \sin ^{2} \theta\right)(1+a) e^{\gamma / \rho}-\left(\rho+\gamma \sin ^{2} \theta\right)(1-a) e^{-\gamma / \rho}\right]}{\rho^{3}\left[(1+a) e^{\gamma / \rho}+(1-a) e^{-\gamma / \rho}\right]^{2}} \\
p_{\varphi} & =\frac{8 \gamma^{2} \sin ^{2} \theta \cos \theta e^{-\lambda}}{\rho^{2}\left[(1+a) e^{\gamma / \rho}+(1-a) e^{-\gamma / \rho}\right]} \\
\mathrm{j}_{t} & =\frac{16 \sqrt{2} \gamma p \cos \theta e^{-\lambda}}{(1+a) \rho^{2}\left[(1+a) e^{\gamma / \rho}+(1-a) e^{-\gamma / \rho}\right]^{3}} \\
\mathrm{j}_{\varphi} & =-\frac{8 \sqrt{2} \gamma q \sin ^{2} \theta e^{-\lambda}}{\rho\left[(1+a) e^{\gamma / \rho}+(1-a) e^{-\gamma / \rho}\right]}
\end{aligned}
$$

In order to study the behavior of these quantities we perform a graphical analysis of them for disks with $\gamma=1$, $z_{0}=1.5$ and $p=q=0,0.5,0.1$, and 1.5. In Fig. 1(a) we show the energy density $\epsilon$ (upper curves) and the azimuthal pressure $p_{\varphi}$ (lower curves), as functions of $r$. We see that the energy density presents a maximum at $r=0$ and then decreases rapidly with $r$. We also see that the presence of electromagnetic field decreases the energy density at the central region of the disk and later increases it. We can observe that the pressure increases rapidly as one moves away from the disk center, reaches a maximum and later decreases rapidly. We also observe that the electromagnetic field decreases the pressure everywhere on the disk. Next, the charge and electric current densities $\mathrm{j}_{t}$ and $\mathrm{j}_{\varphi}$ are represented in Fig. 1 $(b)$. $\mathrm{j}_{t}$ has a maximum at the disk center and then falls to zero at infinity, whereas $\mathrm{j}_{\varphi}$ exhibits a similar behavior to the pressure. We also cumputed the functions $\epsilon, p_{\varphi}, \mathrm{j}_{t}$ and $\mathrm{j}_{\varphi}$ for other values of the parameters and, in all the cases, we found the same behavior.

We now consider the CRM for the same values of the parameters. All the significant quantities can also be expresed in analytic form from the above expressions but the results are so cumbersome that it is best just to analyze them graphically. We first consider the two counterrotating streams circulating along electro-geodesics. In Fig. 2( $a)$ we plot the tangential velocity curves of the counterrotating streams, $\mathrm{U}_{+}$, $\mathrm{U}_{-}$for disks with $\gamma=1, z_{0}=1.5$ and $p=q=0.5$, 0.1 , and 1.5 , and $\mathrm{U}^{2}$ for $\gamma=1, z_{0}=1.5$ and $b=0.5,0.1$, and 1.5 . We can see that $\mathrm{U}_{+}$and $\mathrm{U}_{-}$increase initially and then $U_{+}$falls to zero at infinity and always is less than the light velocity, whereas $U_{-}$increases monotonously. Therefore these disk models are well behaved only at the central regions. However, when the spacetime is electrostatic (or magnetostatic) one finds that the velocity $\mathrm{U}^{2}$ for these disks is always less than the light velocity, but the disks with $b=0.5$ and $z_{0}<0.76$ cannot be built from the CRM because $\mathrm{U}^{2}>1$ (not shown in the figure). One also finds that the inclusion of electric (magnetic) field and the increasing $z_{0}$ make less relativistic these disks.

In Fig. 2(b) we have drawn the specific angular momenta of counterrotating fluids $h_{+}^{2}, h_{-}^{2}$ for disks with $\gamma=1$, $z_{0}=1.5$ and $p=q=0.5,0.1$, and 1.5 , and $h^{2}$ for $\gamma=1, z_{0}=1.5, b=0.5,1.0,1.5$ and 4 . We see that there is a strong change in the slope of $h_{-}^{2}$ at certain value of $r$, which means that there is a strong instability there. We also find regions with negative slope where the CRM is also unstable. Also, $h_{-}^{2}$ presents instability after certain value of r. Therefore these disks models are stable only at the central regions. For electrostatic (or magnetostatic) fields the specific angular momenta $h^{2}$ is an increasing monotonous function of $r$ what corresponds to a stable CRM for the disks. However, the CRM cannot be applied for $b=4.0$ (bottom curve). Thus the inclusion of electric (magnetic) field can make unstable these disks against radial perturbations.

In Fig. 3( $a)$ the plots of the mass densities $\epsilon_{+}, \epsilon_{-}$are shown for disks with $\gamma=1, z_{0}=1.5$ and $p=q=0.5,0.1$, and 1.5, and $\epsilon_{ \pm}$for $\gamma=1, z_{0}=1.5, b=0.5,1.0$, and 1.5, as functions of $r$. The mass density $\epsilon_{+}$is always positive, falling to zero at infinity, whereas $\epsilon_{-}$becomes negative after some value of $r$ and then falls to zero at infinity. The mass densities $\epsilon_{ \pm}$for electrostatic (or magnetostatic) fields are positive everywhere on the disks, falling to zero at infinity. We also see that the presence of electromagnetic field decreases the progradee and retrogade mass densities at the central region of the disks and later increases them.

In Fig. 3 $(b)$ the plots of the electric charge densities $\sigma_{+}, \sigma_{-}$are shown for disks with $\gamma=1, z_{0}=1.5$ and $p=q=0.5$, 0.1 , and 1.5, and $\sigma_{e \pm}$ and $\sigma_{m \pm}$ for $\gamma=1, z_{0}=1.5, b=0.5,1.0$, and 1.5, as functions of $r$. We see that $\sigma_{+}$falls to zero at infinity, whereas $\sigma_{-}$becomes later imaginary. $\sigma_{e \pm}$ and $\sigma_{m \pm}$ present a maximun at the disks center, then decrease monotonously, falling to zero at infinity, and are always real quantities.

As with the electro-geodesic counterrotating fluids we obtain expressions that becomes imaginary in portions of the disks, we also consider non electro-geodesics CRM for these disks. When the two counterrotating streams do not move 
on electro-geodesics but have equal and opposite tangential velocities the graphics for the velocities, energy densities and specific angular momenta of both streams will have the same behavior that in the electrostatic (magnetostatic) case. The expressions for the electric charge densities are differents and are plotted in Fig. 4, being always real quantities. Thus, for Chazy-Curzon-like fields we can build electro-geodesic counterrotating thin disk sources with a well behaved central region, whereas when we have counterrotating streams not moving along electro-geodesic or electrostatic (magnetostatic) fields we obtain physically acceptable counterrotating disk for many values of the parameters.

\section{B. CRM for Zipoy-Voorhees-like disks}

The second family of solutions considered is a Zipoy-Voorhees-like solution which can be written as

$$
\begin{aligned}
e^{\nu} & =\frac{2\left(x^{2}-1\right)^{\gamma / 2}}{(1+a)(x+1)^{\gamma}+(1-a)(x-1)^{\gamma}}, \\
\lambda & =\frac{\gamma^{2}}{2} \ln \left[\frac{x^{2}-1}{x^{2}-y^{2}}\right] \\
A_{t} & =\frac{\sqrt{2} p\left[(x+1)^{\gamma}-(x-1)^{\gamma}\right]}{(1+a)(x+1)^{\gamma}+(1-a)(x-1)^{\gamma}} \\
A_{\varphi} & =\sqrt{2} k \gamma q y,
\end{aligned}
$$

where $a^{2}=1+b^{2}$, with $b^{2}=p^{2}+q^{2}$, and $\gamma$ is a real constant. Here $p$ and $q$ are again the electric and magnetic parameters, respectively. $x$ and $y$ are the prolate spheroidal coordinates, related to the Weyl coordinates by

$$
r^{2}=k^{2}\left(x^{2}-1\right)\left(1-y^{2}\right), \quad z+z_{0}=k x y .
$$

This solution can also be generated, in these coordinates, using the well-known complex potencial formalism proposed by Ernst [31] from the Zipoy-Voorhees vacuum solution [39, 40], also known as the Weyl $\gamma$-solution [41, 42], by choosing the parameter $q$ of Ref. [31] as complex. For $q=0$ we also have an electrostatic solution and for $p=0$ one obtains its magnetostatic equivalent [38]. The case $q=0$ and $\gamma=1$ corresponds to the Reissner-Nordström solution [4], in which case $a=m / k, p=e / k$, with $k^{2}=m^{2}-e^{2}$, so that $a^{2}=1+p^{2}, m$ and $e$ being the mass and charge parameters, respectively. When $p=0$ and $\gamma=2$ one arrives to a Taub-NUT-like magnetostatic solution. This solution can also be generated, in these coordinates, using a well-know theorem proposed by Bonnor (see Ref. [45]) from the Taub-NUT vacuum solution. Note that for $b=0$, it reduces to the Darmois metric [44].

From the above expressions we can compute the physical quantities associated with the disks. We obtain

$$
\begin{aligned}
& \tilde{\epsilon}=\frac{8 \gamma \bar{y}\left(\bar{x}^{2}-1\right)^{\frac{\gamma}{2}(1-\gamma)}\left(\bar{x}^{2}-\bar{y}^{2}\right)^{\frac{1}{2}\left(\gamma^{2}-4\right)}}{\left[(1+a)(\bar{x}+1)^{\gamma}+(1-a)(\bar{x}-1)^{\gamma}\right]^{2}} \\
& \times\left\{\bar{x}\left[(1+a)(\bar{x}+1)^{\gamma}+(1-a)(\bar{x}-1)^{\gamma}\right]\left[\bar{x}^{2}+(\gamma-1) \bar{y}^{2}-\gamma\right]\right. \\
& \left.-\left(\bar{x}^{2}-1\right)\left(\bar{x}^{2}-\bar{y}^{2}\right)\left[(1+a)(\bar{x}+1)^{\gamma-1}+(1-a)(\bar{x}-1)^{\gamma-1}\right]\right\}, \\
& \tilde{p}_{\varphi}=\frac{8 \gamma^{2} \bar{x} \bar{y}\left(1-\bar{y}^{2}\right)\left(\bar{x}^{2}-1\right)^{\frac{1}{2} \gamma(1-\gamma)}\left(\bar{x}^{2}-\bar{y}^{2}\right)^{\frac{1}{2}\left(\gamma^{2}-4\right)}}{\left[(1+a)(\bar{x}+1)^{\gamma}+(1-a)(\bar{x}-1)^{\gamma}\right]} \\
& \tilde{\mathrm{j}}_{t}=\frac{16 \sqrt{2} \gamma p \bar{y}\left(\bar{x}^{2}-1\right)^{\frac{1}{2} \gamma(3-\gamma)}\left(\bar{x}^{2}-\bar{y}^{2}\right)^{\frac{1}{2}\left(\gamma^{2}-2\right)}}{\left[(1+a)(\bar{x}+1)^{\gamma}+(1-a)(\bar{x}-1)^{\gamma}\right]^{3}}, \\
& \mathrm{j}_{\varphi}=-\frac{4 \sqrt{2} \gamma q \bar{x}\left(1-\bar{y}^{2}\right)\left(\bar{x}^{2}-1\right)^{\frac{1}{2} \gamma(1-\gamma)}\left(\bar{x}^{2}-\bar{y}^{2}\right)^{\frac{1}{2}\left(\gamma^{2}-2\right)}}{\left[(1+a)(\bar{x}+1)^{\gamma}+(1-a)(\bar{x}-1)^{\gamma}\right]},
\end{aligned}
$$


where $\tilde{\epsilon}=k \epsilon, \tilde{p}_{\varphi}=k p_{\varphi}$ and $\tilde{\mathrm{j}}_{t}=k \mathrm{j}_{t} . \bar{x}$ and $\bar{y}$ are given by

$$
\begin{aligned}
& 2 \bar{x}=\sqrt{\tilde{r}^{2}+(\alpha+1)^{2}}+\sqrt{\tilde{r}^{2}+(\alpha-1)^{2}}, \\
& 2 \bar{y}=\sqrt{\tilde{r}^{2}+(\alpha+1)^{2}}-\sqrt{\tilde{r}^{2}+(\alpha-1)^{2}},
\end{aligned}
$$

where $\tilde{r}=r / k$ and $\alpha=z_{0} / k$, with $\alpha>1$.

We consider as an example a particular case, when $\gamma=2$. We also study Zipoy-Voorhees-like disks for other values of $\gamma$, but in all cases we found a similar behavior. In Fig. 5 the plots of the quantities $\tilde{\epsilon}, \tilde{p}_{\varphi}, \tilde{\mathrm{j}}_{t}$ and $\mathrm{j}_{\varphi}$ are presented for disks with $\alpha=2.5$ and $p=q=0,0.5,1.0$, and 1.5, as functions of $\tilde{r}$. We see that these functions have a similar behavior to the previous case. Equally, the relevant quantities of the CRM are shown in following figures for the same values of the parameters, also as functions of $\tilde{r}$. We also first consider the two counterrotating streams circulating along electro-geodesics.

Here the tangential velocity $\mathrm{U}_{+}$(Fig. 6(a)) is also always less than the light velocity, falling to zero at infinity, whereas $\mathrm{U}_{-}$is an increasing monotonously function. Thus these disk models are also just well behaved at the central regions. In electrostatic (magnetostatic) case, one also finds that the velocity $\mathrm{U}^{2}$ for these disks is always less than the light velocity, but the disks with $b=0.5$ and $\alpha<1.58$ cannot be constructed from the CRM because $\mathrm{U}^{2}>1$. One also finds that the inclusion of electric (magnetic) field and the increasing $\alpha$ make less relativistic these disks. Moreover, we see that $\tilde{h}_{+}^{2}$ and $\tilde{h}_{-}^{2}$ (Fig. $\left.6(b)\right)$ present instabilities in the same way than the precedent case, and that for electrostatic (or magnetostaic) disks (upper curves) the presence of electric (or magnetic) field can make unstable the CRM against radial perturbations. Thus the CRM cannot apply for $b=4$ (bottom curve).

The mass density $\tilde{\epsilon}_{+}$(Fig. [7 $(a)$ ) is also always positive, falling to zero at infinity, whereas $\tilde{\epsilon}_{-}$becomes negative after some value of $\tilde{r}$ and then falls to zero at infinity. We also see that the presence of electromagnetic field decreases the progradee and retrogade mass densities at the central region of the disks and later increases them. For electrostatic (or magnetostatic) fields $\tilde{\epsilon}_{ \pm}$is positive everywhere on the disks, falling to zero at infinity. We also find that $\tilde{\sigma}_{+}$ (Fig. [7(b)) falls to zero at infinity, whereas $\tilde{\sigma}_{-}$becomes later imaginary. The electrostatic and magnetostatic charge densities, $\tilde{\sigma}_{e \pm}$ and $\tilde{\sigma}_{m \pm}$, are always real quantities, falling to zero at infinity.

Then, when we consider the two counterrotating streams not moving on electro-geodesics but with equal and opposite tangential velocities, one also finds that quantities $\mathrm{U}^{2}, \tilde{\epsilon}_{ \pm}$, and $\tilde{h}^{2}$ have the same behavior than in the electrostatic (magnetostatic) case. The electric charge densities is shown in Fig. 8, being always real quantities. Therefore, for Zipoy-Voorhees-like fields we can build electro-geodesic counterrotating thin disk sources with only a well behaved central region, whereas for counterrotating streams not circulating along electro-geodesic or for electrostatic (magnetostatic) fields, we obtain disks with a physically acceptable CRM for many values of the parameters.

\section{CRM for Bonnor-Sackfield-like disks}

The third family of solutions considered is a Bonnor-Sackfield-like solution which is given by

$$
\begin{aligned}
e^{\nu} & =\frac{2}{(1+a) e^{\gamma \cot ^{-1} u}+(1-a) e^{-\gamma \cot ^{-1} u}}, \\
\lambda & =-\frac{\gamma^{2}}{2} \ln \left[\frac{u^{2}+1}{u^{2}+v^{2}}\right] \\
A_{t} & =\frac{\sqrt{2} p\left[e^{\gamma \cot ^{-1} u}-e^{-\gamma \cot ^{-1} u}\right]}{(1+a) e^{\gamma \cot ^{-1} u}+(1-a) e^{-\gamma \cot ^{-1} u}}, \\
A_{\varphi} & =\sqrt{2} k \gamma q v
\end{aligned}
$$

where $a^{2}=1+b^{2}$, with $b^{2}=p^{2}+q^{2}$, and $\gamma$ is a real constant. Here $p$ and $q$ are again the electric and magnetic parameters, respectively. $u$ and $v$ are the oblate spheroidal coordinates, related to the Weyl coordinates by

$$
r^{2}=k^{2}\left(u^{2}+1\right)\left(1-v^{2}\right), \quad z+z_{0}=k u v .
$$

As in the precedent cases this solution can be generated, in these coordinates, following Ernst's method [31] from the Bonnor-Sackfield vacuum solution [1]. 
From the above expressions we can compute the physical quantities associated with the disks. We obtain

$$
\begin{aligned}
& \tilde{\epsilon}=\frac{8 \gamma \bar{v}\left(\bar{u}^{2}+1\right)^{\frac{\gamma^{2}}{2}}}{(\bar{u}+\bar{v})^{\frac{\gamma^{2}}{2}+2}\left[(1+a) e^{\gamma \cot ^{-1} \bar{u}}+(1-a) e^{-\gamma \cot ^{-1} \bar{u}}\right]^{2}} \\
& \times\left\{\left[\bar{u}^{2}+\bar{v}^{2}-\gamma \bar{u}\left(1-\bar{v}^{2}\right)\right](1+a) e^{\gamma \cot ^{-1} \bar{u}}-\left[\bar{u}^{2}+\bar{v}^{2}+\gamma \bar{u}\left(1-\bar{v}^{2}\right)\right](1-a) e^{-\gamma \cot ^{-1} \bar{u}}\right\} \\
& \tilde{p}_{\varphi}=\frac{8 \gamma^{2} \bar{u} \bar{v}\left(1-\bar{v}^{2}\right)(\bar{u}+1)^{\frac{\gamma^{2}}{2}}}{(\bar{u}+\bar{v})^{\frac{\gamma^{2}}{2}+2}\left[(1+a) e^{\gamma \cot ^{-1} \bar{u}}+(1-a) e^{-\gamma \cot ^{-1} \bar{u}}\right]}, \\
& \tilde{\mathrm{j}}_{t}=\frac{16 \sqrt{2} \gamma p \bar{v}(\bar{u}+1)^{\frac{\gamma^{2}}{2}}}{(\bar{u}+\bar{v})^{\frac{\gamma^{2}}{2}+1}\left[(1+a) e^{\gamma \cot ^{-1} \bar{u}}+(1-a) e^{-\gamma \cot ^{-1} \bar{u}}\right]^{3}}, \\
& \mathrm{j}_{\varphi}=-\frac{4 \sqrt{2} \gamma q \bar{u}\left(1-\bar{v}^{2}\right)(\bar{u}+1)^{\gamma^{2} / 2}}{(\bar{u}+\bar{v})^{\frac{\gamma^{2}}{2}+1}\left[(1+a) e^{\gamma \cot ^{-1} \bar{u}}+(1-a) e^{-\gamma \cot ^{-1} \bar{u}}\right]},
\end{aligned}
$$

where $\tilde{\epsilon}=k \epsilon, \tilde{p}_{\varphi}=k p_{\varphi}$ and $\tilde{\mathrm{j}}_{t}=k \mathrm{j}_{t} . \bar{u}$ and $\bar{v}$ are given by

$$
\begin{aligned}
& \sqrt{2} \bar{u}=\sqrt{\left[\left(\tilde{r}^{2}+\alpha^{2}-1\right)^{2}+4 \alpha^{2}\right]^{1 / 2}+\left(\tilde{r}^{2}+\alpha^{2}-1\right)}, \\
& \sqrt{2} \bar{v}=\sqrt{\left[\left(\tilde{r}^{2}+\alpha^{2}-1\right)^{2}+4 \alpha^{2}\right]^{1 / 2}-\left(\tilde{r}^{2}+\alpha^{2}-1\right)},
\end{aligned}
$$

where $\tilde{r}=r / k$ and $\alpha=z_{0} / k$.

In Figs. 9] 12 the plots of the physical quantities related with the disks are presented for disks with $\gamma=1, \alpha=1.5$ and $p=q=0,0.5,1.0$, and 1.5, also as functions of $\tilde{r}$. We see that these functions have the same behavior that the previous cases. Therefore, when we consider counterrotating streams moving along electro-geodesics only the central region of the disks is well behaved, whereas in the case of counterrotating streams not circulating along electrogeodesics or for electrostatic (magnetostatic) fields we obtain disks with a physically acceptable CRM for many values of the parameters.

\section{CRM for Kerr-like disks}

Finally, other family of solutions to the Einstein-Maxwell equations, is a Kerr-like solution which is given by

$$
\begin{aligned}
\nu & =\ln \left[\frac{a^{2} x^{2}-b^{2} y^{2}-1}{(a x+1)^{2}-b^{2} y^{2}}\right] \\
\lambda & =2 \ln \left[\frac{a^{2} x^{2}-b^{2} y^{2}-1}{a^{2}\left(x^{2}-y^{2}\right)}\right], \\
A_{t} & =\frac{2 \sqrt{2} p y}{(a x+1)^{2}-b^{2} y^{2}} \\
A_{\varphi} & =-\frac{\sqrt{2} k q\left(1-y^{2}\right)(a x+1)}{a\left(a^{2} x^{2}-b^{2} y^{2}-1\right)}
\end{aligned}
$$

where $a^{2}=1+b^{2}$, with $b^{2}=p^{2}+q^{2}$. Here $p$ and $q$ are also the electric and magnetic parameters, respectively. $x$ and $y$ are again the prolate spheroidal coordinates, given by (37). For $b=0$ this solution also reduces to the Darmois metric. Moreover, for $q=0$ we have a Kerr-like electrostatic solution. This solution can be generated, in these coordinates, using a well-know theorem proposed by Bonnor (see Ref. [45]) from the Kerr vacuum solution. And for $p=0$ one obtains its magnetostatic equivalent. This solution is asymptotically flat and was firts studied by Bonnor [46] in a different system of coordinates and describes the field of a massive magnetic dipole. 
The physical quantities associated with the disks can now be written as

$$
\begin{aligned}
\tilde{\epsilon}= & \frac{8 a^{4} \bar{y}}{\left(a^{2} \bar{x}^{2}-b^{2} \bar{y}^{2}-1\right)^{2}\left[(a \bar{x}+1)^{2}-b^{2} \bar{y}^{2}\right]^{2}} \\
& \times\left\{\left(\bar{x}^{2}-\bar{y}^{2}\right)\left[a\left(\bar{x}^{2}-1\right)\left[(a \bar{x}+1)^{2}+b^{2} \bar{y}^{2}\right]-2 b^{2} \bar{x}(a \bar{x}+1)\left(1-\bar{y}^{2}\right)\right]\right. \\
& \left.\quad-2 \bar{x}\left(\bar{x}^{2}-1\right)\left(1-\bar{y}^{2}\right)\left[(a \bar{x}+1)^{2}-b^{2} \bar{y}^{2}\right]\right\} \\
\tilde{p}_{\varphi}= & \frac{16 a^{4} \bar{x} \bar{y}\left(\bar{x}^{2}-1\right)\left(1-\bar{y}^{2}\right)}{\left(a^{2} \bar{x}^{2}-b^{2} \bar{y}^{2}-1\right)^{2}\left[(a \bar{x}+1)^{2}-b^{2} \bar{y}^{2}\right]}, \\
\tilde{\mathrm{j}}_{t}= & -\frac{4 \sqrt{2} a^{4} p\left(\bar{x}^{2}-\bar{y}^{2}\right)\left\{\bar{x}\left(1-\bar{y}^{2}\right)\left[(a \bar{x}+1)^{2}+b^{2} \bar{y}^{2}\right]-2 a \bar{y}^{2}(a \bar{x}+1)\left(\bar{x}^{2}-1\right)\right\}}{\left(a^{2} \bar{x}^{2}-b^{2} \bar{y}^{2}-1\right)\left[(a \bar{x}+1)^{2}-b^{2} \bar{y}^{2}\right]^{3}}, \\
\mathrm{j}_{\varphi}= & -\frac{2 \sqrt{2} a^{4} q \bar{y}\left(\bar{x}^{2}-1\right)\left(1-\bar{y}^{2}\right)\left(\bar{x}^{2}-\bar{y}^{2}\right)\left[(a \bar{x}+1)(3 a \bar{x}+1)+b^{2} \bar{y}^{2}\right]}{\left(a^{2} \bar{x}^{2}-b^{2} \bar{y}^{2}-1\right)^{3}\left[(a \bar{x}+1)^{2}-b^{2} \bar{y}^{2}\right]},
\end{aligned}
$$

where $\bar{x}$ and $\bar{y}$ are given by Eqs. (39a) and (39b).

In figs. 13, 16 the plots of the physical quantities describing the disks are shown for $\alpha=1.7$ and $p=q=0,0.4$, 0.7 , and 1.5, as functions of $\tilde{r}$. The energy density behaves in the opposite way to the previous cases. That is, near to the disk center it increases when the electromagnetic field is applied and decreases later. The other quantities have a similar behavior to the earlier cases. However, the electric charge density jo after some $\tilde{r}$ takes negative values. Likewise, the quantities corresponding to the CRM are shown in following figures for the same values of the parameters, also as functions of $\tilde{r}$. Again, we first consider the two counterrotating streams circulating along electro-geodesics.

Now the tangential velocities $\mathrm{U}_{+}$and $\mathrm{U}_{-}$are always less than the light velocity, falling to zero at infinity (Fig. 14 $(a))$. Note moreover that the two counterrotating streams initially circulate with different velocities and then become equal and opossite. Thus these disks models are well behaved everywhere on the disks. We also found that for some values of the parameters cannot be constructed disks from the CRM because $\mathrm{U}^{2}>1$. We also note that the presence of electromagnetic field can stabilize the CRM against radial perturbations (Fig. 14 (b)). On the other hand, the mass densities $\tilde{\epsilon}_{+}, \tilde{\epsilon}_{-}$and $\tilde{\epsilon}_{ \pm}$(Fig. 15 $(a)$ ) are also always positive and fall to zero at infinity.

In Fig. 15(b) we have plotted the charge densities $\tilde{\sigma}_{+}, \tilde{\sigma}_{-}, \sigma_{e \pm}$ and $\sigma_{m \pm}$. We see that $\tilde{\sigma}_{+}$and $\sigma_{m \pm}$ are always positive, falling to zero at infinity, whereas $\tilde{\sigma}_{-}$can take initially negative values and $\sigma_{e \pm}$ become negative after some value of $\tilde{r}$ and then fall to zero at infinity. Thus, the Kerr-like disk with $\alpha=1.7$ and $p=q=1.5$ can be constructed from the counterrotating streams circulating along electro-geodesics. Finally, the electric charge densities when the two streams do not move on electro-geodesics are shown in Fig. 16, being always real quantities. Therefore, in all the cases considered we can build Kerr-like disks with a phisically acceptable CRM for many values of the parameters.

\section{DISCUSSION}

A detailed study of the counterrotating model for generic electrovacuum static axially symmetric relativistic thin disks without radial pressure was presented. A general constraint over the counterrotating tangential velocities was found, needed to cast the surface energy-momentum tensor of the disk in such a way that it can be interpreted as the superposition of two counterrotating charged dust fluids. The constraint found is completely equivalent to the necessary and sufficient condition obtained in reference [33]. We next showed that this constraint is satisfied if we take the two counterrotating fluids as circulating along electro-geodesics. We note that en general the two fluids circulate with different velocities. However, when the spacetime is electrostatic (or magnetostatic) the two geodesic fluids circulate with equal and opposite velocities. We also have obtained explicit expressions for the energy densities, current densities and velocities of the counterrotating streams in terms of the energy density, azimuthal pressure and planar current density of the disks, that are also equivalent to the correspondig expressions in reference [33].

Four families of models of counterrotating charged disks were considered in the present work based in simple solutions to the vacumm Einsteins-Maxwell equations in the static axisymmetric case generated by conventional solutiongenerating tecniques [44]. When the two counterrotating streams move on electro-geodesisc, Chazy-Curzon-like, Zipoy-Voorhees and Bonnor-Sackfield-like counterrotating disks can be constructed only with a well behaved central region, whereas for Kerr-like fields we found for many values of the parameters disks with a physically acceptable CRM. On the other hand, when we consider the two counterrotating fluids not circulating on electro-geodesic but with 
equal and opposite velocities or for electrostatic (or magnetostatic) fields, we found for many values of the parameter disks with a well behaved CRM everywhere. In the case of Chazy-Curzon-like, Zipoy-Voorhees and Bonnor-Sackfieldlike fields, we also saw that the presence of electric (magnetic) field can make unstable against radial perturbations the CRM, and conversely, stabilizes the CRM in the case of Kerr-like disks.

Finally, the generalization of the counterrotating model presented here to the case with radial pressure is in consideration. Also, the generalization to rotating thin disks with or without radial pressure in presence of electromagnetic fields is being considered.

\section{Acknowledgments}

Gonzalo García R. wants to thank a Fellowship from Vicerrectoría Académica, Universidad Industrial de Santander.

[1] W. A. Bonnor and A. Sackfield, Comm. Math. Phys. 8, 338 (1968).

[2] T. Morgan and L. Morgan, Phys. Rev. 183, 1097 (1969).

[3] L. Morgan and T. Morgan, Phys. Rev. D2, 2756 (1970).

[4] A. Chamorro, R. Gregory and J. M. Stewart, Proc. R. Soc. Lond. A 413, 251 (1987).

[5] G. A. González and P. S. Letelier. Class. Quantum. Grav. 16, 479 (1999).

[6] D. Lynden-Bell and S. Pineault, Mon. Not. R. Astron. Soc. 185, 679 (1978).

[7] P.S. Letelier and S. R. Oliveira, J. Math. Phys. 28, 165 (1987).

[8] J. P. S. Lemos, Class. Quantum Grav. 6, 1219 (1989).

[9] J. P. S. Lemos and P. S. Letelier, Class. Quantum Grav. 10, L75 (1993).

[10] J. Bičák, D. Lynden-Bell and J. Katz, Phys. Rev. D47, 4334 (1993).

[11] J. Bičák, D. Lynden-Bell and C. Pichon, Mon. Not. R. Astron. Soc. 265, 126 (1993).

[12] J. Bičák and T. Ledvinka. Phys. Rev. Lett. 71, 1669 (1993).

[13] J. P. S. Lemos and P. S. Letelier, Phys. Rev D49, 5135 (1994).

[14] J. P. S. Lemos and P. S. Letelier, Int. J. Mod. Phys. D5, 53 (1996).

[15] C. Klein, Class. Quantum Grav. 14, 2267 (1997).

[16] G. A. González and P. S. Letelier. Phys. Rev. D 62, 064025 (2000).

[17] V. C. Rubin, J. A. Graham and J. D. P Kenney. Ap. J. 394, L9-L12, (1992).

[18] H. Rix, M. Franx, D. Fisher and G. Illingworth. Ap. J. 400, L5-L8, (1992).

[19] T. Ledvinka, J. Bičák, and M. Žofka, in Procceeding of 8th Marcel-Grossmann Meeting in General Relativity, edited by T. Piran (Worls Scientific, Singapure, 1999)

[20] P. S. Letelier. Phys. Rev. D 60, 104042 (1999).

[21] J. Katz, J. Bičák and D. Lynden-Bell, Class. Quantum Grav. 16, 4023 (1999).

[22] J. L. Synge, Relativity: The General Theory. (North Holland, 1966)

[23] G. Neugebauer and R. Meinel, Phys. Rev. Lett. 75, 3046 (1995).

[24] C. Klein, Class. Quantum Grav. 14, 2267 (1997).

[25] C. Klein and O. Richter, Phys. Rev. Lett. 83, 2884 (1999).

[26] C. Klein, Phys. Rev. D63, 064033 (2001).

[27] J. Frauendiener and C. Klein, Phys. Rev. D63, 084025 (2001).

[28] C. Klein, Phys. Rev. D65, 084029 (2002).

[29] C. Klein, Phys. Rev. D68, 027501 (2003).

[30] G. A. González and O. A. Espitia, Relativistic Static Thin Disks: The counterrotating model. To appear in Phys. Rev. D.

[31] F. J. Ernst. Phys. Rev. D 168, 1415 (1968).

[32] P. S. Letelier. Phys. Rev. D 22, 807 (1980).

[33] J. J. Ferrando, J. A. Morales and M. Portilla. Gen. Rel. and Grav. 22, 1021 (1990).

[34] S. Chandrasekar, The Mathematical Theory of Black Holes. (Oxford University Press, 1992).

[35] L. D. Landau and E. M. Lifshitz, Fluid Mechanics (Addisson-Wesley, Reading, MA, 1989).

[36] Chazy J 1924 Bull. Soc. Math., France 5217

[37] Curzon H E J 1924 Proc. London Math. Soc. 23477

[38] W. B. Bonnor. Proc. Phys. Soc. Lond. A 67, 225 (1954).

[39] D. M. Zipoy. J. Math. Phys. 7, 1137 (1966)

[40] B. H. Voorhees. Phys. Rev. D 2, 2119 (1970)

[41] H. Weyl. Ann. Phys. 54, 117 (1917)

[42] H. Weyl. Ann. Phys. 59, 185 (1919)

[43] K. Schwarzschild. Sitzungsber. Preuss. Akad. Wiss., 189 (1916).

[44] D. Kramer, H. Stephani, E. Herlt, and M. McCallum, Exact Solutions of Einsteins's Field Equations (Cambridge University Press, Cambridge, England, 1980). 


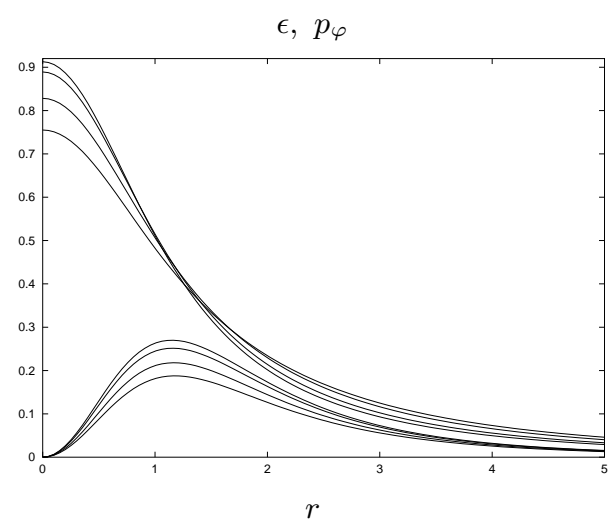

$(a)$

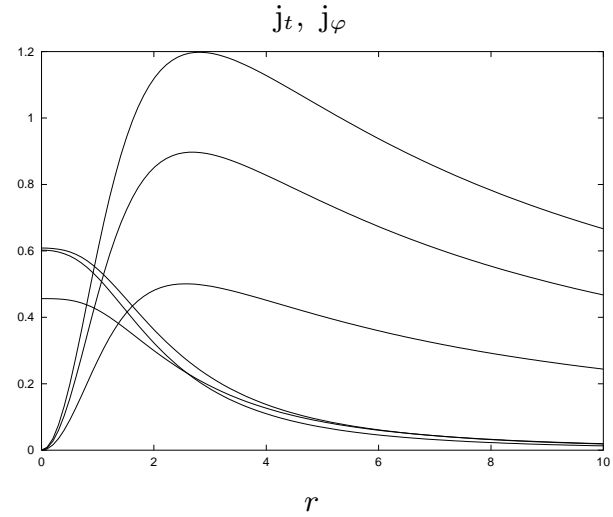

$(b)$

FIG. 1: $(a) \epsilon$ (upper curves) and $p_{\varphi}$ (scaled by a factor of 2.5), as functions of $r$ for Chazy-Curzon-like disks with $\gamma=1$, $z_{0}=1.5$ and $p=q=0$ (top curves), $0.5,1.0$, and 1.5 (bottom curves). (b) $\mathrm{j}_{t}$ (lower curves scaled by a factor of 20 ) and $\mathrm{j}_{\varphi}$ for the same values of the parameters.

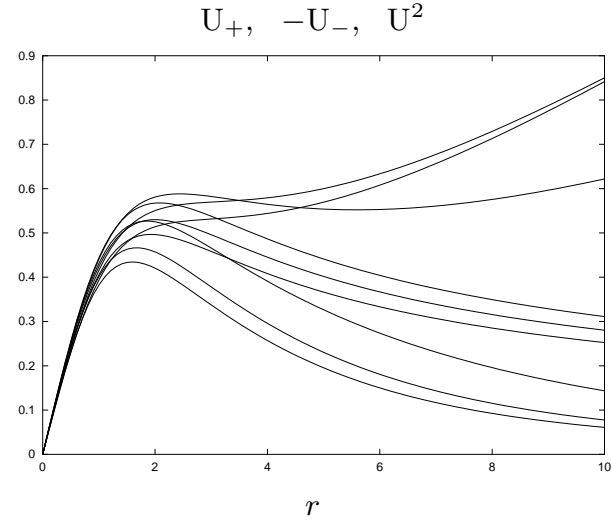

(a)

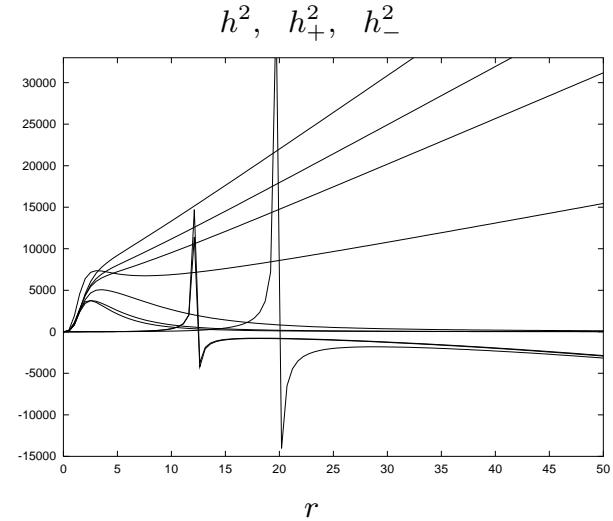

$(b)$

FIG. 2: (a) $\mathrm{U}_{+}$(lower curves), $\mathrm{U}_{-}$(upper curves) for Chazy-Curzon-like disks with $\gamma=1, z_{0}=1.5$ and $p=q=0.5,1.0$, and 1.5, and $\mathrm{U}^{2}$ for $\gamma=1, z_{0}=1$ and $b=0.5,1.0$, and 1.5. (b) $h_{+}^{2}$ (scaled by a factor of 1000), $h_{-}^{2}$ (sharp curves ) for the same values of the parameters and $h^{2}$ (upper curves scaled by a factor of 1000) for $\gamma=1, z_{0}=1.5$ and $b=0.5,1.0,1.5$, and 4 (bottom curve).

[45] W. B. Bonnor. Z. Phys. 161, 439 (1961).

[46] W. B. Bonnor. Z. Phys. 190, 444 (1966). 


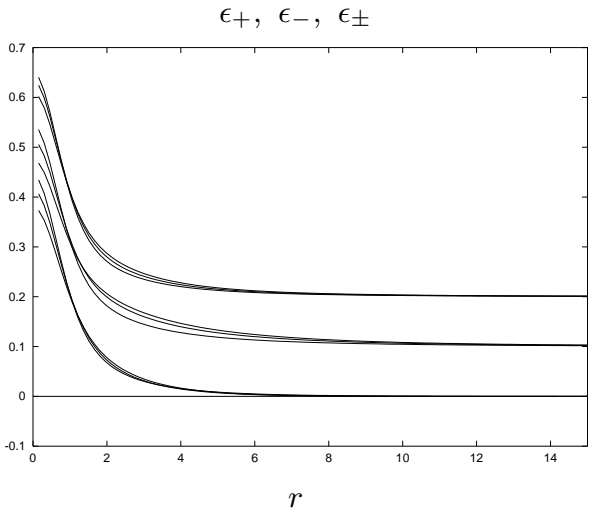

(a)

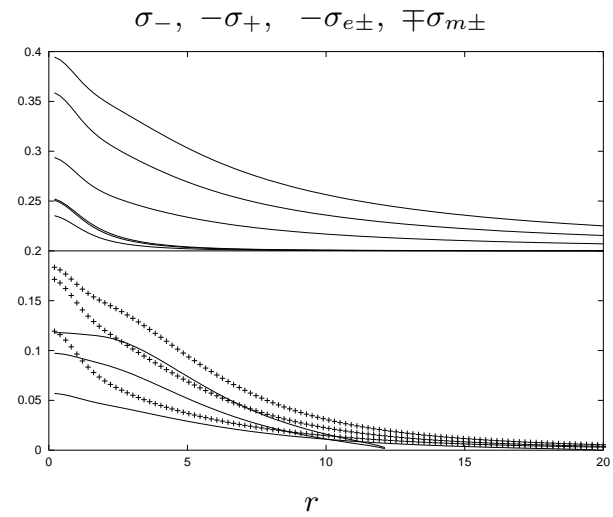

$(b)$

FIG. 3: (a) $\epsilon_{-}$(lower curves), $\epsilon_{+}$(moved upwards a factor of 0.1) for Chazy-Curzon-like disks with $\gamma=1, z_{0}=1.5$ and $p=q=0.5,1.0$, and 1.5 and $\epsilon_{ \pm}$(upper curves moved upwards a factor of 0.2 ) for $\gamma=1, z_{0}=1.5$ and $b=0.5,1.0$ and 1.5 . (b) $\sigma_{-}, \sigma_{+}$(curves with crosses), $\sigma_{e \pm}$ (moved upwards a factor of 0.2) and $\sigma_{m \pm}$ (upper curves moved also upwards a factor of $0.2)$ for the same values of the parameters.

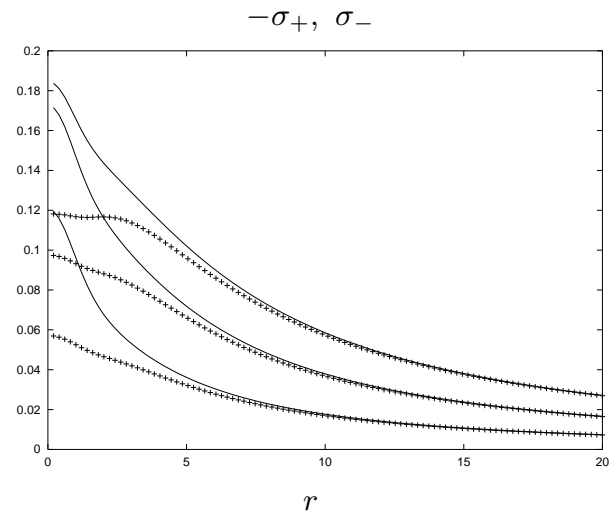

FIG. 4: $\sigma_{+}$(solid curves) and $\sigma_{-}$for not electro-geodesic Chazy-Curzon-like disks with $\gamma=1, z_{0}=1.5$ and $p=q=0.5$ (lower curves), 1.0, and 1.5 (upper curves). 


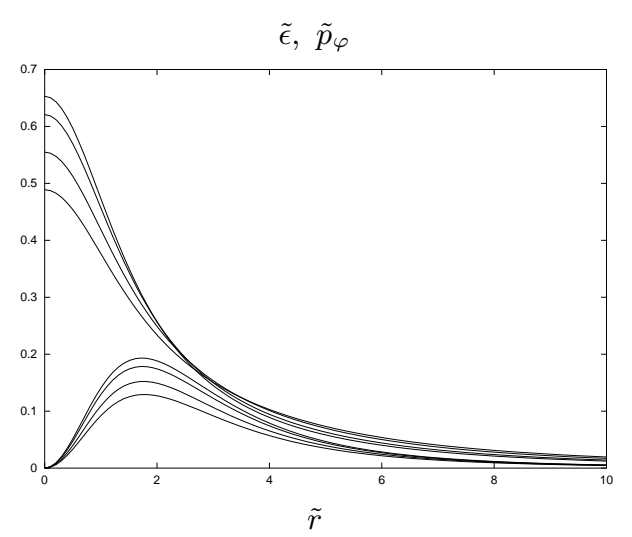

$(a)$

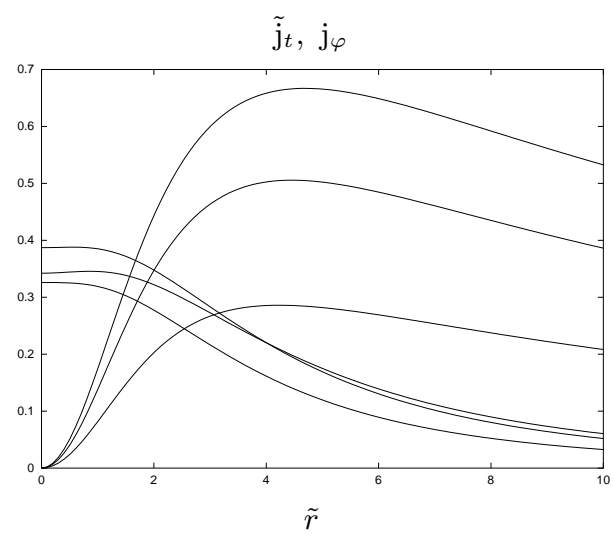

$(b)$

FIG. 5: (a) $\tilde{\epsilon}$ (upper curves ) and $\tilde{p}_{\varphi}$ (scaled by a factor of 2), as functions of $\tilde{r}$ for Zipoy-Voorhees-like disks with $\gamma=2, \alpha=2.5$ and $p=q=0$ (top curves), 0.5, 1.0, and 1.5 (bottom curves). (b) $\tilde{\mathrm{j}}_{t}$ (lower curves scaled by a factor of 10 ) and $\mathrm{j}_{\varphi}$ for the same values of the parameters.

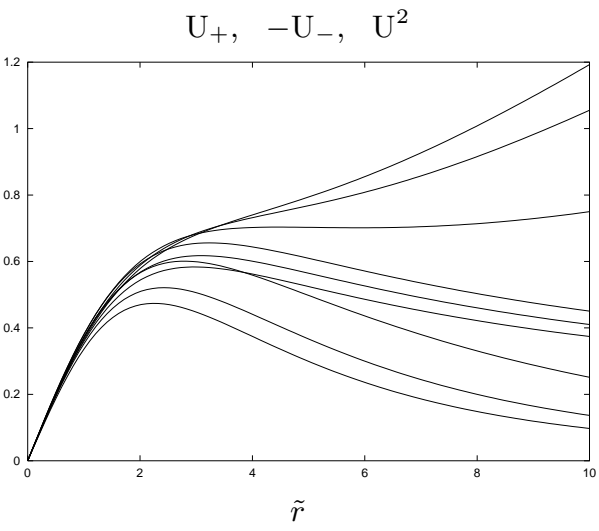

(a)

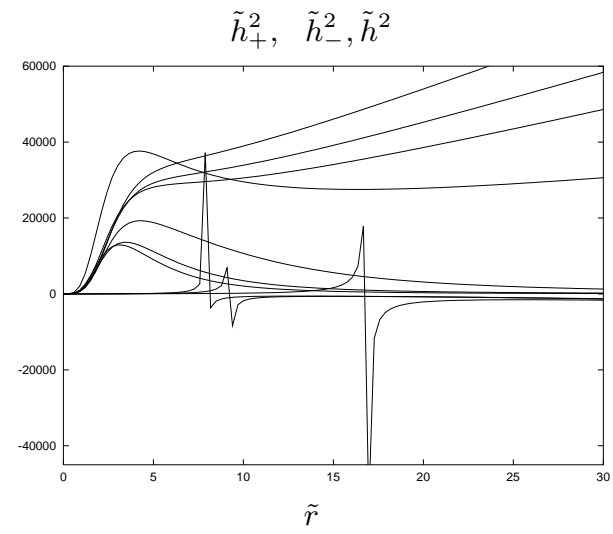

(b)

FIG. 6: (a) $\mathrm{U}_{+}$(lower curves), $\mathrm{U}_{-}$(upper curves) as functions of $\tilde{r}$ for Zipoy-Voorhees-like disks with $\gamma=2, \alpha=2.5$ and $p=q=0.5,1.0$, and 1.5 and $\mathrm{U}^{2}$ for $\gamma=2, \alpha=2.5$ and $b=0.5,1.0$, and 1.5 . (b) $\tilde{h}_{+}^{2}$ (scaled by a factor of 1000), $\tilde{h}_{-}^{2}$ (sharp curves) for the same values of the parameters and $\tilde{h}^{2}$ (upper curves scaled by a factor of 1000) for $\gamma=2, \alpha=2.5$ and $b=0.5$, 1.0, 1.5 and 4.0 (bottom curve). 


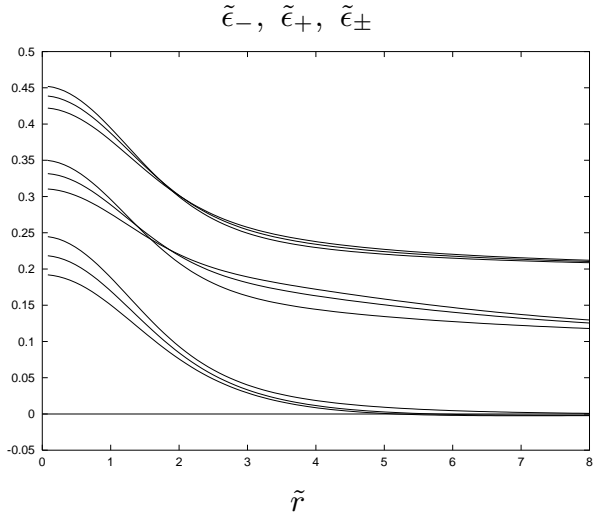

(a)

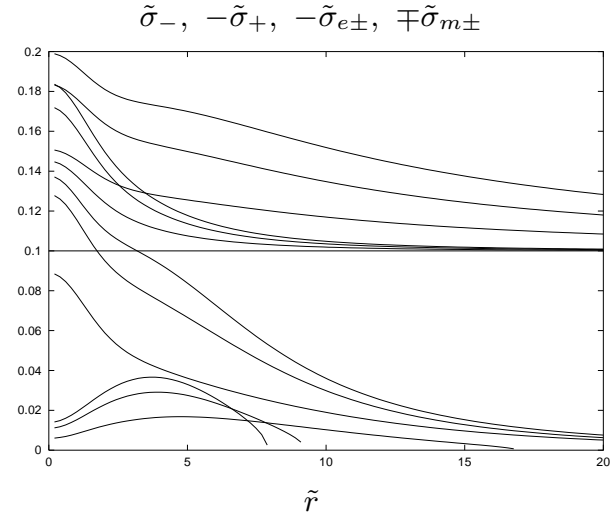

$(b)$

FIG. 7: (a) $\tilde{\epsilon}_{-}$(lower curves), $\tilde{\epsilon}_{+}$(moved upwards a factor of 0.1 ) for Zipoy-Voorhees-like disks with $\gamma=2, \alpha=2.5$ and $p=q=0.5,1.0$, and 1.5 and $\tilde{\epsilon}_{ \pm}$(upper curves moved upwards a factor of 0.2 ) for $\gamma=2, \alpha=2.5$ and $b=0.5,1.0$ and 1.5 . (b) $\tilde{\sigma}_{-}$(lower curves), $\tilde{\sigma}_{+}, \tilde{\sigma}_{e \pm}$ (moved upwards a factor of 0.1 ) and $\tilde{\sigma}_{m \pm}$ (upper curves moved also upwards a factor of 0.1 ) for the same values of the parameters.

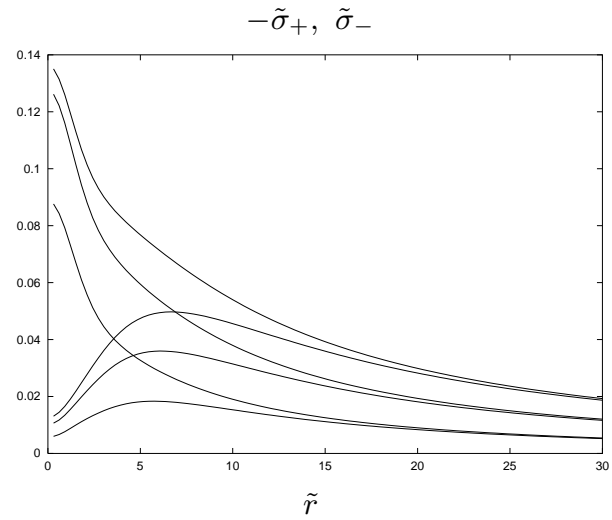

FIG. 8: $\tilde{\sigma}_{+}$(upper curves) and $\tilde{\sigma}_{-}$for not electro-geodesic Zipoy-Voorhees-like disks with $\gamma=2, \alpha=2.5$ and $p=q=0.5$ (lower curves), 1.0, and 1.5 (upper curves).

$\tilde{\epsilon}, \tilde{p}_{\varphi}$

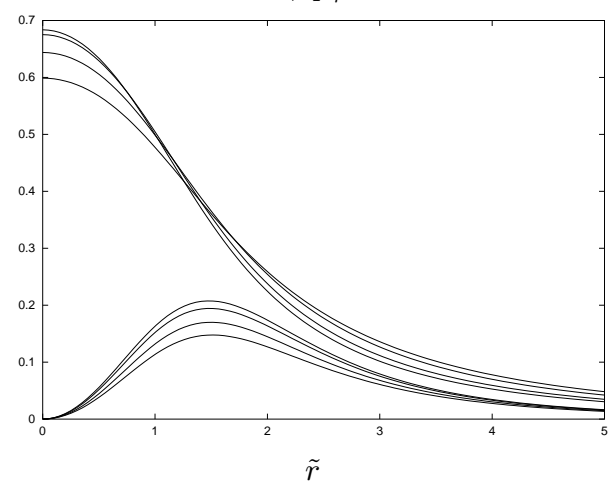

(a) $\tilde{\mathrm{j}}_{t}, \mathrm{j}_{\varphi}$

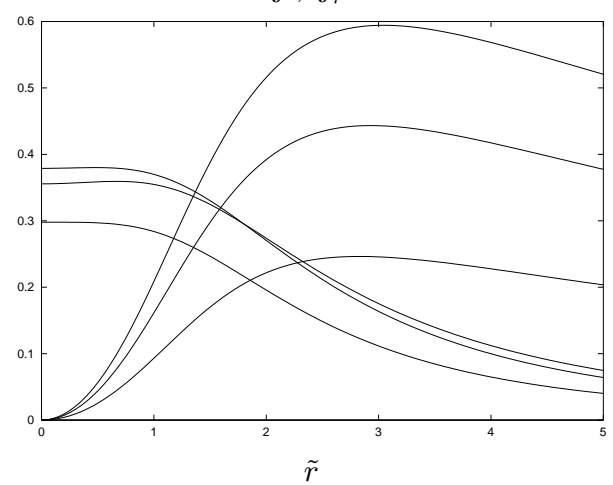

(b)

FIG. 9: (a) $\tilde{\epsilon}$ (upper curves ) and $\tilde{p}_{\varphi}$ (scaled by a factor of 2.5), as functions of $\tilde{r}$ for Bonnor-Sackfield-like disks with $\gamma=1$, $\alpha=1.5$ and $p=q=0$ (top curves), $0.5,1.0$, and 1.5 (bottom curves). (b) $\tilde{\mathrm{j}}_{t}$ (lower curves scaled by a factor of 5 ) and $\mathrm{j}_{\varphi}$ for the same values of the parameters. 


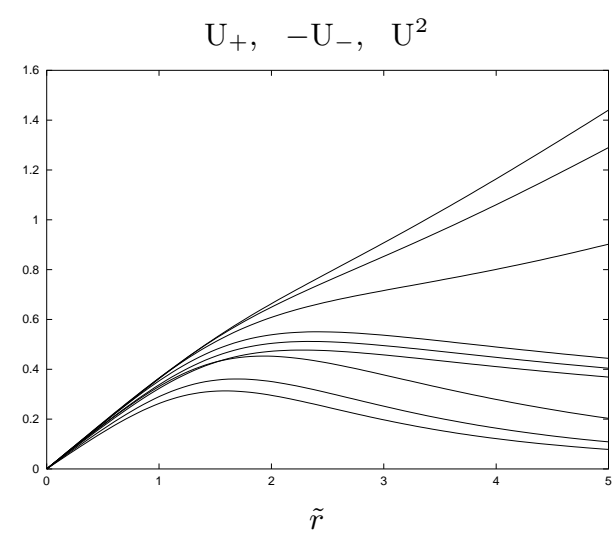

(a)

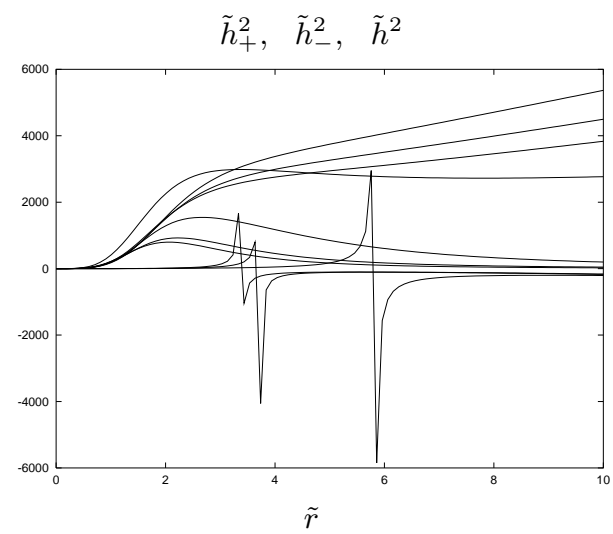

(b)

FIG. 10: (a) $\mathrm{U}_{+}$(lower curves), $\mathrm{U}_{-}$(upper curves) for Bonnor-Sackfield-like disks with $\gamma=1, \alpha=1.5$ and $p=q=0.5,1.0$, and 1.5 and $\mathrm{U}^{2}$ for $\gamma=1, \alpha=1.5$ and $b=0.5,1.0$, and 1.5, as functions of $\tilde{r}$. (b) $\tilde{h}_{+}^{2}$ (scaled by a factor of 500), $\tilde{h}_{-}^{2}$ (sharp curves) for the same values of the parameters and $\tilde{h}^{2}$ (upper curves scaled by a factor of 400 ) for $\gamma=1, \alpha=1.5$ and $b=0.5$, $1.0,1.5$ and 4.0 (bottom curve).

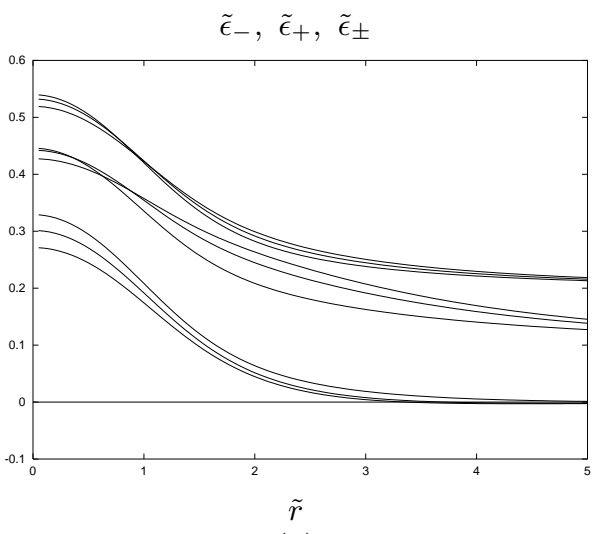

(a)

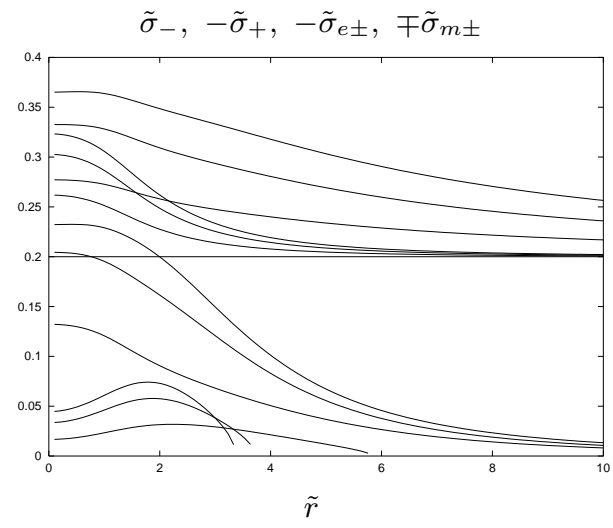

(b)

FIG. 11: (a) $\tilde{\epsilon}_{-}$(lower curves), $\tilde{\epsilon}_{+}$(moved upwards a factor of 0.1) for Bonnor-Sackfield-like disks with $\gamma=1, \alpha=1.5$ and $p=q=0.5,1.0$, and 1.5 and $\tilde{\epsilon}_{ \pm}$(upper curves moved upwards a factor of 0.2 ) for $\gamma=1, \alpha=1.5$ and $b=0.5,1.0$ and 1.5 . (b) $\tilde{\sigma}_{-}$(lower curves), $\tilde{\sigma}_{+}, \tilde{\sigma}_{e \pm}$ (moved upwards a factor of 0.2) and $\tilde{\sigma}_{m \pm}$ (upper curves moved also upwards a factor of 0.2) for the same values of the parameters. 


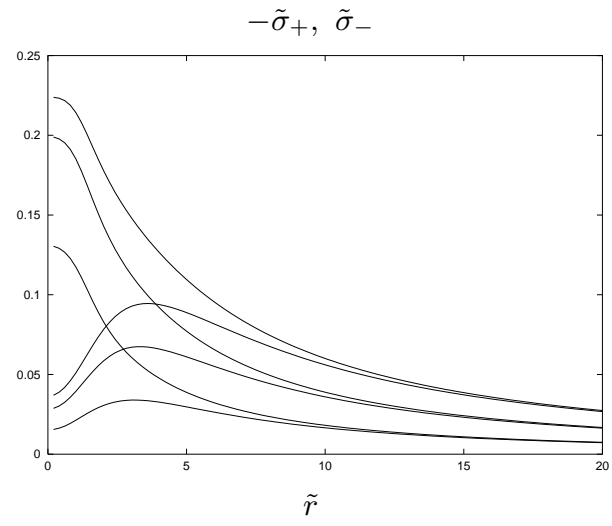

FIG. 12: $\tilde{\sigma}_{+}$(upper curves) and $\tilde{\sigma}_{-}$for not electro-geodesic Bonnor-Sackfield-like disks with $\gamma=1, \alpha=1.5$ and $p=q=0.5$ (lower curves), 1.0, and 1.5 (upper curves).

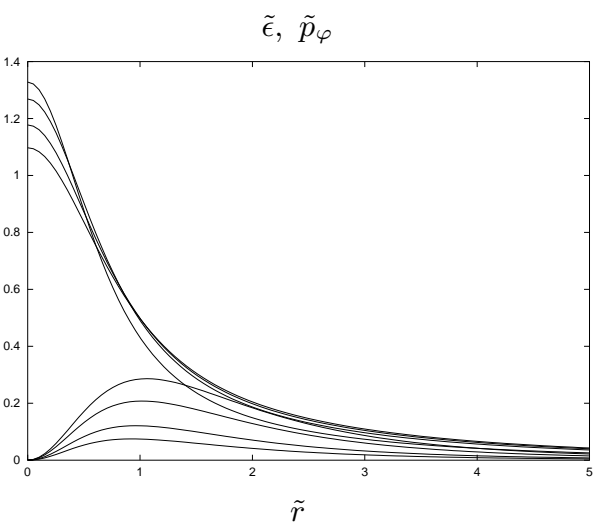

(a)

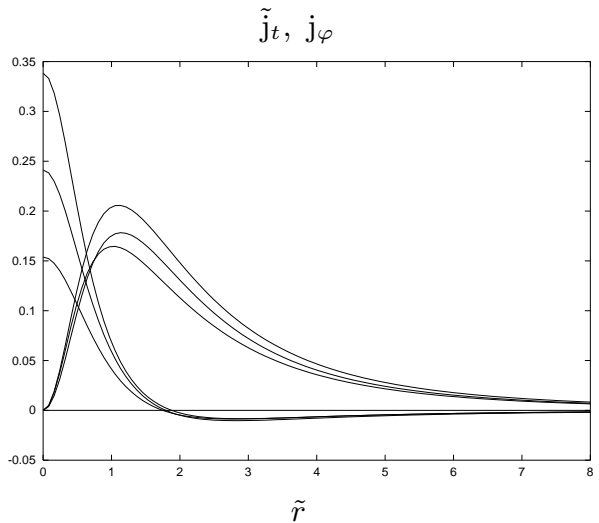

(b)

FIG. 13: (a) $\tilde{\epsilon}$ (lower curves ) and $\tilde{p}_{\varphi}$, as functions of $\tilde{r}$ for Kerr-like disks with $\alpha=1.7$ and $p=q=0$ (bottom and top curves), $0.4,0.7$, and 1.5 (top and bottom curves). (b) $\tilde{\mathrm{j}}_{t}$ (upper curves ) and $\mathrm{j}_{\varphi}$ for the same values of the parameters.

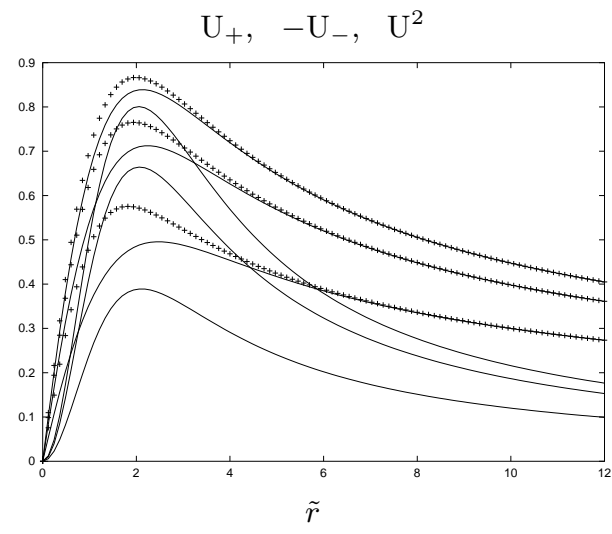

$(a)$

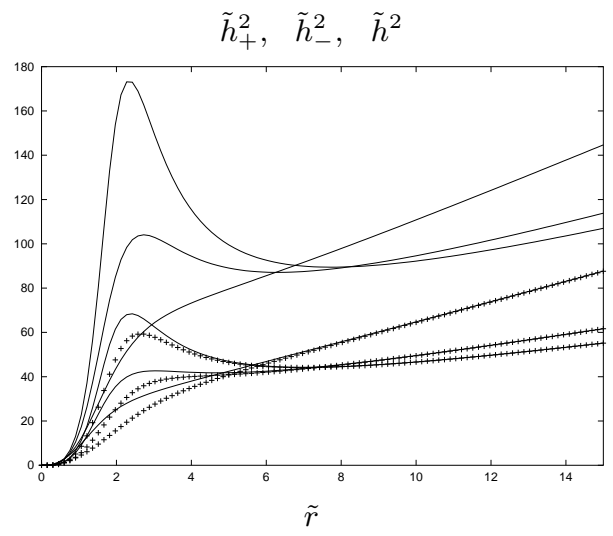

(b)

FIG. 14: (a) $\mathrm{U}_{+}$and $\mathrm{U}_{-}$(curves with crosses) for Kerr-like disks with $\alpha=1.7$ and $p=q=0.4,0.7$, and 1.5 , and $\mathrm{U}^{2}$ for $\alpha=1.7$ and $b=0.4,0.7,1.5$, as functions of $\tilde{r}$. (b) $\tilde{h}_{+}^{2}$ (curves with crosses), $\tilde{h}_{-}^{2}$ for the same values of the parameters and $\tilde{h}^{2}$ (upper curves scaled by a factor of 2 ) for $\alpha=1.7$ and $b=0.4,0.7,1.5$ and 4.0 (bottom curve). 


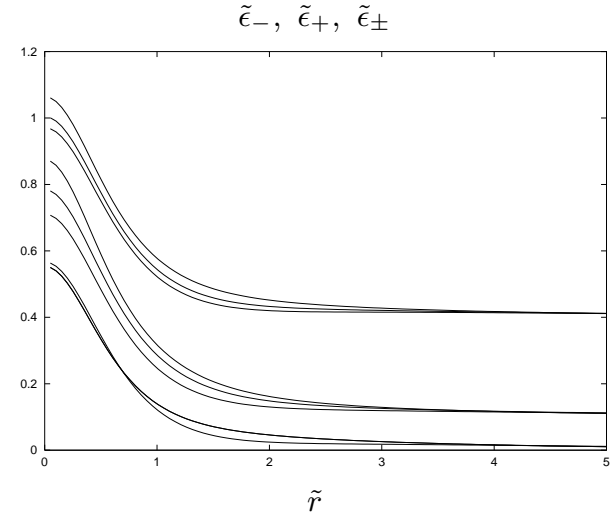

$(a)$

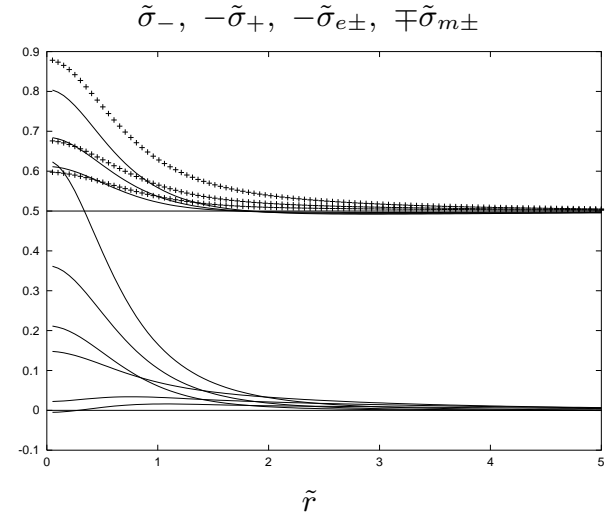

$(b)$

FIG. 15: (a) $\tilde{\epsilon}_{-}$(lower curves), $\tilde{\epsilon}_{+}$(moved upwards a factor of 0.1) for Kerr-like disks with $\alpha=1.7$ and $p=q=0.4,0.7$, and 1.5 and $\tilde{\epsilon}_{ \pm}$(upper curves moved upwards a factor of 0.4 ) for $\alpha=1.7$ and $b=0.4,0.7$ and 1.5. (b) $\tilde{\sigma}_{-}$(lower curves), $\tilde{\sigma}_{+}, \tilde{\sigma}_{e \pm}$ (moved upwards a factor of 0.5) and $\tilde{\sigma}_{m \pm}$ (curves with crosses moved also upwards a factor of 0.5) for the same values of the parameters.

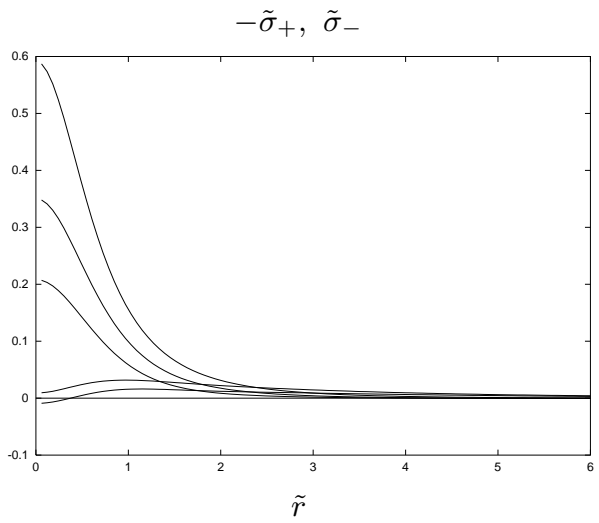

FIG. 16: $\tilde{\sigma}_{+}$(upper curves) and $\tilde{\sigma}_{-}$for not electro-geodesic Kerr-like disks with $\alpha=1.7$ and $p=q=0.4$ (lower curves), 0.7 , and 1.5 (upper curves). 